\title{
Fusion in Multibiometric Identification Systems: What about the Missing Data?^
}

\author{
Karthik Nandakumar ${ }^{1}$, Anil K. Jain ${ }^{2}$, and Arun Ross ${ }^{3}$ \\ 1 Institute for Infocomm Research, A*STAR, Fusionopolis, Singapore \\ knandakumar@i2r.a-star.edu.sg \\ 2 Michigan State University, East Lansing, MI, USA \\ jainacse.msu.edu \\ 3 West Virginia University, Morgantown, WV, USA \\ arun.ross@mail.wvu.edu
}

\begin{abstract}
Many large-scale biometric systems operate in the identification mode and include multimodal information. While biometric fusion is a well-studied problem, most of the fusion schemes have been implicitly designed for the verification scenario and cannot account for missing data (missing modalities or incomplete score lists) that is commonly encountered in multibiometric identification systems. In this paper, we show that likelihood ratio-based score fusion, which was originally designed for verification systems, can be extended for fusion in the identification scenario under certain assumptions. We further propose a Bayesian approach for consolidating ranks and a hybrid scheme that utilizes both ranks and scores to perform fusion in identification systems. We also demonstrate that the proposed fusion rules can handle missing information without any ad-hoc modifications. We observe that the recognition performance of the simplest rank level fusion scheme, namely, the highest rank method, is comparable to the performance of complex fusion strategies, especially when the goal is not to obtain the best rank-1 accuracy but to just retrieve the top few matches.
\end{abstract}

\section{Introduction}

Biometric systems can operate in two modes, namely, verification and identification. In verification, the query is compared only to the template of the claimed identity and the identity claim is accepted as "genuine" if the degree of similarity is sufficiently high. On the other hand, the goal in identification systems is to determine the identity of an individual from a large set of possible identities. Here, the user's biometric input is compared with the templates of all the persons enrolled in the database and the system outputs either the identity of the person whose template has the highest degree of similarity with the user's input (closed set ID) or a decision indicating that the user presenting the input is not an enrolled user (open set ID). Many large-scale biometric applications such as the FBI-IAFIS and US-VISIT IDENT program work in the openset identification mode. Since the number of enrolled users in the database can be quite

\footnotetext{
* This research was supported by the Center for Identification Technology Research at West Virginia University.
} 
large (e.g., more than 80 million subjects in the FBI-IAFIS system [1]), the identification task is significantly more challenging than verification.

Since multibiometric systems provide several advantages over unimodal systems such as increased population coverage and lower error rates, the databases in many large scale ID systems are increasingly becoming multimodal (e.g., fingerprint, face and palmprint in Next Generation IAFIS, multiple fingers and face in US-VISIT). Biometric fusion has been extensively studied in the literature and a number of fusion schemes have been proposed [2]. However, most of the existing fusion schemes have been implicitly designed for the verification scenario. For instance, the density-based and classifier-based approaches typically model biometric fusion as a two-class (genuine and impostor) classification problem. Hence, such schemes cannot be directly applied to the identification scenario. In [3], Tulyakov and Govindaraju identified four possible strategies in the context of combining multi-class classifiers and described a method for improving the verification performance of a biometric system by utilizing scores obtained by matching the query to all the templates in the database. However, they fail to provide a technique for optimizing the identification performance.

Moreover, the problem of missing data is frequently encountered in large scale multibiometric ID systems. The missing information may be in the form missing modalities in the template or the query or incomplete score/rank information from the individual matchers. In many pattern classification systems, the problem of missing feature values is handled either through imputation (predicting the missing values based on the available features) or by designing dynamic classification rules. One such example is the approach proposed by Dinerstein et al. [4], which involves learning a separate Support Vector Machine (SVM) classifier for each possible combination of input modalities and selecting the appropriate SVM based on the query presented during authentication. However, such a solution is clearly inefficient and not scalable.

Our goal is to develop a biometric fusion scheme specifically for the identification mode that can seamlessly handle partial information without any need for designing rules on a case-by-case basis. Towards this end, we have made the following three contributions. Firstly, we present a mathematical framework for score fusion in identification systems. Secondly, we propose a Bayesian scheme for rank-level fusion in multibiometric systems, which can be combined with the score fusion framework to obtain a hybrid score-rank fusion scheme. Finally, we also demonstrate the effectiveness of the proposed schemes in handling missing information.

\section{Score Fusion in Multibiometric Identification}

A likelihood ratio (LR)-based framework for score level fusion was presented in [5] for the verification scenario. This LR framework is based on the Neyman-Pearson theorem and it maximizes the genuine accept rate (GAR) at any desired false accept rate (FAR), provided the genuine and impostor match densities are known or can be estimated accurately. By following a Bayesian approach, we now show that the likelihood ratios computed in the LR framework can also be used for fusion in the identification scenario provided the following three assumptions are satisfied: (i) prior probabilities are equal for all users, (ii) the match scores for different persons are independent of one another, and (iii) genuine (impostor) match scores of all users are identically distributed. 
Table 1. Transpose of a sample match score matrix, $\boldsymbol{S}$, from the NIST-Multimodal database. Since the number of enrolled users in this database is 517 and the number of modalities is $4, S$ is a $517 \times 4$ matrix.

\begin{tabular}{|c|c|c|c|c|c|c|c|c|c|}
\hline User \# & 1 & 2 & 3 & 4 & $\cdots$ & 514 & 515 & 516 & 517 \\
\hline Left Index Finger & 29 & 17 & 8 & 8 & $\cdots$ & 9 & 6 & 4 & 9 \\
\hline Right Index Finger & 84 & 7 & 7 & 5 & $\cdots$ & 8 & 5 & 4 & 7 \\
\hline Face Matcher 1 & 0.58 & 0.57 & 0.46 & 0.51 & $\cdots$ & 0.52 & 0.55 & 0.49 & 0.52 \\
\hline Face Matcher 2 & 73.6 & 73.9 & 65.7 & 65.4 & $\cdots$ & 66.3 & 66.7 & 66.8 & 72.2 \\
\hline
\end{tabular}

Let $K$ denote the number of matchers in the multibiometric system and $N$ be the number of persons enrolled in the system. Suppose for a given query, we observe the $N \times K$ score matrix $\boldsymbol{S}=\left[s_{n}^{k}\right]$ (see Table 1). Note that $s_{n}^{k}$ represents the match score output by the $k^{t h}$ matcher for the $n^{t h}$ template in the database, $k=1, \cdots, K ; n=$ $1, \cdots, N$. Our goal is to determine the true identity $I$ of the given query based on $S$. According to the Bayes decision theory [6], the query should be assigned to the identity $I_{n_{0}}$ that maximizes the posteriori probability, i.e.,

$$
\begin{gathered}
\text { Assign query to identity } I_{n_{0}} \text { if } \\
P\left(I_{n_{0}} \mid \boldsymbol{S}\right) \geq P\left(I_{n} \mid \boldsymbol{S}\right), \forall n=1, \cdots, N .
\end{gathered}
$$

The above decision rule applies only to closed set identification. For open set identification, the query is assigned to identity $I_{n_{0}}$ only when equation (1) holds and $P\left(I_{n_{0}} \mid \boldsymbol{S}\right) \geq$ $\tau$, where $\tau$ is a threshold. We can estimate the posteriori probabilities $P\left(I_{n} \mid \boldsymbol{S}\right)$ in the following manner. According to the Bayes theorem,

$$
P\left(I_{n} \mid \boldsymbol{S}\right)=\frac{p\left(\boldsymbol{S} \mid I_{n}\right) P\left(I_{n}\right)}{p(\boldsymbol{S})},
$$

where $p\left(\boldsymbol{S} \mid I_{n}\right)$ is the likelihood of observing the score matrix $\boldsymbol{S}$ given that the true identity is $I_{n}$ and $P\left(I_{n}\right)$ is the prior probability of observing the identity $I_{n}$. Under the assumption of equal priors for all users, the posteriori probability $P\left(I_{n} \mid \boldsymbol{S}\right)$ is proportional to the likelihood $p\left(\boldsymbol{S} \mid I_{n}\right)$. Ideally, we would like to estimate the conditional density of $\boldsymbol{S}$ individually for each user because it captures the complete information about dependencies between the scores assigned to the different users and the user-specific characteristics of the match scores. However, directly estimating the conditional density of $\boldsymbol{S}$ is not practical due to the following two reasons: (i) since $\boldsymbol{S}$ is a $N \times K$ dimensional matrix and $N$ is usually quite large in identification scenarios, estimating the density of $\boldsymbol{S}$ requires a significant number of training samples for each user, which is not generally available in multibiometric databases; and (ii) the density of $\boldsymbol{S}$ needs to be re-estimated frequently due to changes in the list of enrollees.

If match scores for different persons are independent of one another, the likelihood $p\left(\boldsymbol{S} \mid I_{n}\right)$ can be simplified as

$$
p\left(\boldsymbol{S} \mid I_{n}\right)=\prod_{j=1}^{N} p\left(\boldsymbol{s}_{j} \mid I_{n}\right)=p\left(\boldsymbol{s}_{n} \mid I_{n}\right) \prod_{j=1, j \neq n}^{N} p\left(\boldsymbol{s}_{j} \mid I_{n}\right) .
$$


Here, $p\left(s_{n} \mid I_{n}\right)$ represents the density of genuine match scores corresponding to user $I_{n}$ (denoted by $\left.f_{\text {gen, }, n}(\cdot)\right)$ and $p\left(s_{j} \mid I_{n}\right), j \neq n$ represents the densities of the impostor scores (denoted by $f_{i m p, n}(\cdot)$ ). However, when the genuine (impostor) match scores of all users are assumed to be identically distributed $\left(f_{\text {gen }, n}(\cdot)=f_{\text {gen }}(\cdot)\right.$ and $f_{i m p, n}(\cdot)=$ $\left.f_{\text {imp }}(\cdot), \forall n\right)$, equation (3) can be further simplified as

$$
p\left(\boldsymbol{S} \mid I_{n}\right)=f_{\text {gen }}\left(\boldsymbol{s}_{n}\right) \prod_{j=1, j \neq n}^{N} f_{i m p}\left(\boldsymbol{s}_{j}\right)=\frac{f_{\text {gen }}\left(\boldsymbol{s}_{n}\right)}{f_{\text {imp }}\left(\boldsymbol{s}_{n}\right)} \prod_{j=1}^{N} f_{i m p}\left(\boldsymbol{s}_{j}\right) .
$$

Thus, the likelihood of observing the score matrix $S$ given that the true identity is $I_{n}$ is proportional to the likelihood ratio that was used by the authors in [5] for the verification scenario. Furthermore, if we assume that the scores of different matchers are conditionally independent, we can estimate the joint density of the genuine (impostor) match scores by the product of the marginal densities. Hence, the decision rule in equation (1) can be restated as

$$
\begin{gathered}
\text { Assign query to identity } I_{n_{0}} \text { if } \\
\prod_{k=1}^{K} \frac{f_{g e n}^{k}\left(s_{n_{0}}^{k}\right)}{f_{i m p}^{k}\left(s_{n_{0}}^{k}\right)} \geq \prod_{k=1}^{K} \frac{f_{g e n}^{k}\left(s_{n}^{k}\right)}{f_{i m p}^{k}\left(s_{n}^{k}\right)}, \forall n=1, \cdots, N,
\end{gathered}
$$

where $f_{g e n}^{k}(\cdot)$ and $f_{i m p}^{k}(\cdot)$ are the marginal densities of the genuine and impostor match scores, respectively, output by the $k^{\text {th }}$ matcher.

\section{Bayesian Approach for Rank Level Fusion}

When a biometric system operates in the identification mode, the output of the system may also be a set of ranks (possible matching identities sorted in a decreasing order of match scores). Although the ranks are derived from the match scores, the rank information captures the relative ordering of the scores corresponding to different users. Suppose for a given query, we observe the $N \times K$ rank matrix $\boldsymbol{R}=\left[r_{n}^{k}\right]$, where $r_{n}^{k}$ represents the rank output by the $k^{t h}$ matcher for the $n^{\text {th }}$ template in the database, $k=1, \cdots, K ; n=1, \cdots, N$. Let $r_{n}^{\prime}$ be a statistic computed for user $n$ such that the user with the lowest value of $r^{\prime}$ is assigned the highest consensus (or reordered) rank. For example, in the highest rank method [7], each user is assigned the highest rank (minimum $r$ value) as computed by different matchers, i.e., the statistic for user $n$ is

$$
r_{n}^{\prime}=\min _{k=1}^{K} r_{n}^{k}
$$

Ties are broken randomly to arrive at a strict ranking order based on the new statistic $r^{\prime}$. Ho et al. [7] proposed other methods such as Borda Count and logistic regression, which compute the statistic $r^{\prime}$ as a linear combination of the ranks. Melnik et al. [8] proposed the use of non-linear functions to combine the ranks of the individual matchers.

We now propose a new rank combination statistic based on Bayes decision theory. Let $P_{k}(r)$ be the probability that the identity which is assigned rank $r$ by the $k^{t h}$ 
matcher is the true identity, $r=1, \cdots, N ; k=1, \cdots, N$. Note that the cumulative distribution function of the discrete rank distribution $P_{k}(r)$ is nothing but the Cumulative Match Characteristic (CMC) curve [9], which is commonly used to depict the performance of an identification system. Grother and Phillips [10] and Bolle et al. [11] show that the rank distribution $P_{k}(r)$ can be estimated provided the marginal genuine and impostor match score densities $f_{g e n}^{k}(\cdot)$ and $f_{i m p}^{k}(\cdot)$ are known. This estimation again requires two of the assumptions used in section 2 namely, (i) scores of the individual users are independent and (ii) score distributions of different users are identical.

For a given query, suppose that the identity $I_{n}$ is assigned the rank $r_{n}^{k}$ by the $k^{t h}$ matcher. From the definition of the rank distribution $P_{k}(r), P_{k}\left(r_{n}^{k}\right)$ is the posteriori probability that $I_{n}$ is the true identity given $r_{n}^{k}$. Further, if we assume that the matchers are independent, we can compute the new rank combination statistic as the product of the posterior probabilities of the individual matchers.

$$
r_{n}^{\prime}=\prod_{k=1}^{K} P_{k}\left(r_{n}^{k}\right), \text { for } n=1, \cdots, N .
$$

For the rank statistic computed using equation (7), the user with the largest value of $r^{\prime}$ should be assigned the highest consensus rank. So, the rank posterior based fusion rule can then be defined as follows.

$$
\begin{aligned}
& \text { Assign query to identity } I_{n_{0}} \text { if } \\
& r_{n_{0}}^{\prime} \geq r_{n}^{\prime}, \forall n=1,2, \cdots, N .
\end{aligned}
$$

Note that likelihood ratio based score fusion rule shown in equation (5) utilizes only the match scores corresponding to a particular user, when computing the likelihood ratio for that user. In other words, the relative information between the scores of different users is ignored when computing the score likelihood ratio. On the other hand, the rank posterior based fusion rule in equation (8) considers only the relative order information between the scores of different users and the actual score values are ignored. Therefore, we can treat the score and rank information as two different pieces of evidence and define a hybrid fusion scheme that utilizes both the match scores and the ranks. Let $R$ be the combined score and rank statistic, defined as

$$
R_{n}(\boldsymbol{S}, \boldsymbol{R})=P\left(I_{n} \mid \boldsymbol{S}\right) r_{n}^{\prime}
$$

where the posterior probability based on the match score matrix $\boldsymbol{S}, P\left(I_{n} \mid \boldsymbol{S}\right)$, is computed by substituting equation (4) in equation (2) and the posterior probability based on the rank matrix $\boldsymbol{R}$ is obtained using equation (7). The hybrid score-rank fusion rule can then be defined as

$$
\begin{aligned}
& \text { Assign query to identity } I_{n_{0}} \text { if } \\
& R_{n_{0}} \geq R_{n}, \forall n=1,2, \cdots, N \text {. }
\end{aligned}
$$




\section{Fusion with Missing Data}

In a multibiometric ID system, three types of missing data can occur. Firstly, not all the biometric information corresponding to an individual may be available in the database. This leads to missing modalities (templates) for some of the users. Secondly, all the modalities may not be available from the user during authentication, resulting in incomplete queries. Finally, the individual matchers may output a partial list of scores or ranks corresponding to only the top $m$ matching identities. Missing data needs to be accounted for, both when training the fusion algorithm and during identification.

The likelihood ratio-based score fusion technique presented in equation (5) has the ability to handle the three kinds of missing data. Since we assume that the score distributions are identical for all users and the matchers are independent, we need to estimate only the marginal genuine and impostor match score densities for each matcher. Therefore, even if some of the scores in the score matrix $S$ are not available for a fraction of the users, we can still estimate the required densities by pooling match scores from all the users. During identification, when a match score $s_{n}^{k}$ is not available, it implies that the $k^{t h}$ matcher does not provide any evidence in support of the $n^{t h}$ identity. This fact can be easily incorporated in equation (5) by setting the corresponding likelihood ratio $f_{g e n}^{k}\left(s_{n}^{k}\right) / f_{i m p}^{k}\left(s_{n}^{k}\right)$ to the value 1 with no change in the decision rule required.

Missing data affects the rank posterior-based fusion scheme more severely. When there are missing templates in the database, the rank information provided by a matcher is not quite meaningful. An incorrect identity may be assigned rank 1 (even when the match score is very low) if the template corresponding to the true identity is not available. Hence, it is not possible to estimate the rank distribution accurately when there are missing templates. Consequently, the rank posterior-based fusion scheme and the hybrid score-rank fusion scheme cannot be employed in this scenario. However, in the case of a missing modality in the query sample presented during authentication, the rank posterior-based technique can still be used by assigning equal posterior probabilities to all the users, i.e., if the $k^{t h}$ modality is not available in the query sample, $P\left(I_{n} \mid r_{n}^{k}\right)=1 / N, \forall n=1, \cdots, N$. Partial rank lists can be handled by randomly assigning lower ranks to the identities that were not originally assigned a rank by the matcher. For example, if a matcher outputs only the ranks corresponding to the top $m$ matching identities, the other identities can be randomly assigned the ranks from $(m+1)$ to $N$. In most biometric matchers, the probability $P_{k}(r)$ is high only for the top few matches and is very small for large $r$. Therefore, the random assignment of lower ranks does not affect the performance of the system.

\section{Performance Evaluation}

The identification performances of various score and rank level fusion strategies were evaluated on the three partitions of the NIST-BSSR1 database. The first partition is the NIST-Multimodal database, consisting of 517 users with two fingerprints (left and right index fingers) and two face scores (obtained from two different face matchers). The second partition of NIST-BSSR1 is the NIST-Fingerprint database, which is an example of multi-instance(finger) biometric system. This partition consists of scores 




(a)

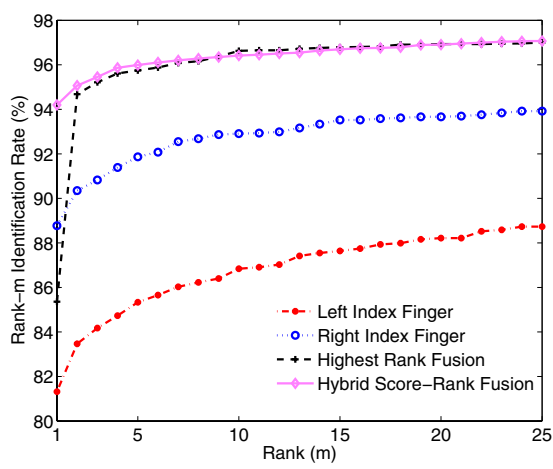

(b)

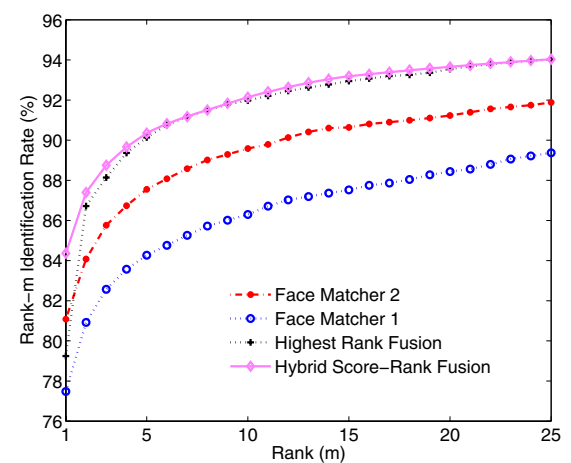

(c)

Fig. 1. Cumulative Match Characteristic (CMC) curve of highest rank fusion and the hybrid scorerank fusion rules on (a) NIST-Multimodal ( $K=4, N=517$ ), (b) NIST-Fingerprint ( $K=$ $2, N=6,000)$ and (c) NIST-Face $(K=2, N=3,000)$ databases. The CMC curves shown here are the average recognition rates obtained over 20 cross-validation trials.

from left and right index fingerprint matches of 6,000 individuals. The third partition is the NIST-Face database, which consists of scores from two face matchers applied on three frontal face images from 3,000 individuals.

The cumulative match characteristic (CMC) curves of the individual matchers and the highest rank and hybrid score-rank fusion rules on the NIST-BSSR1 database are shown in Figures 1(a), 1(b) and 1(c). In each experiment, half the users were randomly selected to be in the training set for estimating the marginal densities and the rank distribution. The remaining half of the database was used for evaluating the fusion performance. The above training-test partitioning was repeated 20 times and the reported CMC curves correspond to the mean identification rates over the 20 trials. The Gaussian mixture model based scheme [5] was used for estimating the score densities.

Among the various rank level fusion schemes such as highest rank, Borda count and logistic regression, we observed that the highest rank method achieves the best rank-m 
recognition rate when $m \geq K$, where $K$ is the number of matchers. Hence, only the recognition rates of the highest rank method are reported here. It is well-known that the highest rank method works well when the number of users is large compared to the number of matchers [7], which is usually the case in biometric identification systems. This is because the highest rank method utilizes the strength of each matcher effectively. Even if only one matcher assigns a high rank to the correct user, it is likely that the correct user will receive a high rank after reordering. However, there can be up to $K$ ties at rank 1 due to conflicting decisions output by the $K$ matchers. Since the ties are broken randomly without considering the relative accuracies of the matchers, the identification rates at ranks 1 to $K-1$ are not very high. In fact, the rank-1 accuracy of the highest rank method is usually less than that of the best individual matcher.

The recognition rates of likelihood ratio-based score fusion, rank posterior fusion and hybrid score-rank fusion rules were observed to be quite similar on all the three partitions of NIST-BSSR1. While the hybrid score-rank fusion rule achieves a marginal improvement in the recognition rates over the other two fusion rules, the differences in the recognition rates of the three fusion rules is less than $1 \%$ at all ranks. Therefore, only the performance of the hybrid score-rank fusion rule is reported in Figures 1(a), 1(b) and 1(c) In the case of the NIST-Multimodal database, the hybrid score-rank fusion rule provides $100 \%$ rank-1 accuracy, while the rank-1 accuracy of the best single matcher (right index finger) was only $93.7 \%$. The hybrid score-rank fusion rule improves the rank-1 accuracy from $88.9 \%$ for the best single matcher (right index finger) to $94 \%$ on the NIST-Fingerprint database. Finally, on the NIST-Face database the improvement is comparatively lower $(81.2 \%$ for the best face matcher and $84.1 \%$ for the score-rank fusion rule) due to the strong correlation between the two face matchers.

The results also indicate that the performance of the simplest rank level fusion scheme, namely, the highest rank method, is comparable to performance of the more complex score and rank fusion strategies for ranks greater than or equal to $K$, where $K$ is the number of matchers. Therefore, in practical multibiometric ID systems with a large number of users, it may be sufficient to use the highest rank method if the goal is to retrieve the top few matches. However, if the best rank-1 accuracy is desired and if the score information is available, then the hybrid score-rank fusion rule can be employed.

We have also evaluated the robustness of the fusion schemes under the missing data scenario. We simulate missing data by randomly removing scores from the score matrices obtained from NIST-BSSR 1 with probability $m_{p}$ (whose value is set to $0.05,0.1$ or $0.25)$. When the template corresponding to the $k^{t h}$ modality of user $n$ is deemed to be missing, only the score $s_{n}^{k}$ is removed. When the $k^{t h}$ modality is deemed to be missing in the query, the $k^{t h}$ column in the score matrix $\boldsymbol{S}$ is removed (see Table 2). While simulating missing data, care is taken to ensure that each user has a template corresponding to at least one modality and each query provides at least one modality to be used for identification. To avoid cases where there are no common modalities between the query and the user templates in the database (e.g., only the left index fingerprint of a user is available in the database, whereas the query contains only the right index fingerprint) we study the missing template and missing query scenarios separately.

Figure 2] shows the CMC curves for different fusion strategies under the missing data scenario on the NIST-Face database (similar results were observed for the other two 
Table 2. Transpose of a sample match score matrix with missing data (denoted by $\mathrm{x}$ ) simulated from the NIST-Multimodal database. In this example, the query multimodal sample does not contain the fingerprint from the left index finger, the templates corresponding to the right index finger are not available for users 3 and 517 and face templates are not available for user 515 .

\begin{tabular}{|c|c|c|c|c|c|c|c|c|c|}
\hline User \# & 1 & 2 & 3 & 4 & $\cdots$ & 514 & 515 & 516 & 517 \\
\hline Left Index Finger & $\mathbf{x}$ & $\mathbf{x}$ & $\mathbf{x}$ & $\mathbf{x}$ & $\cdots$ & $\mathbf{x}$ & $\mathbf{x}$ & $\mathbf{x}$ & $\mathbf{x}$ \\
\hline Right Index Finger & 84 & 7 & $\mathbf{x}$ & 5 & $\cdots$ & 8 & 5 & 4 & $\mathbf{x}$ \\
\hline Face Matcher 1 & 0.58 & 0.57 & 0.46 & 0.51 & $\cdots$ & 0.52 & $\mathbf{x}$ & 0.49 & 0.52 \\
\hline Face Matcher 2 & 73.6 & 73.9 & 65.7 & 65.4 & $\cdots$ & 66.3 & $\mathbf{x}$ & 66.8 & 72.2 \\
\hline
\end{tabular}

databases). As explained in section 4 the rank-posterior and hybrid score-rank fusion schemes cannot be applied in the case of missing templates. Only the likelihood ratio based score fusion rule is used in this scenario (see figure 2(a)). On the other hand, the hybrid score-rank fusion rule can be used in the missing query modalities scenario (see figure 2(b)]. Similar to the case, where is there is no missing data, the performance of the highest rank fusion rule is comparable to that of the score fusion rules for larger ranks. However, the highest rank fusion rule has a significantly worse rank-1 recognition rate in the case of missing templates, because the ranks assigned by the matchers are not very meaningful when there are missing templates.

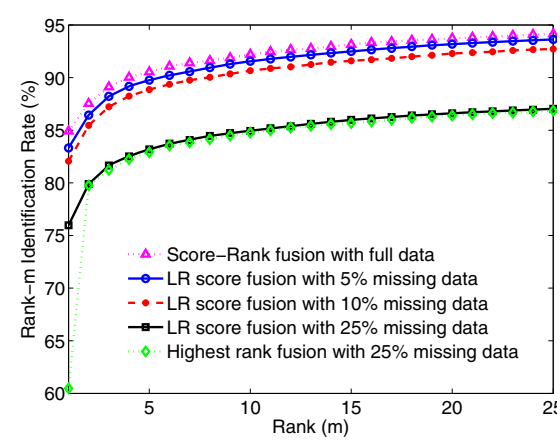

(a)

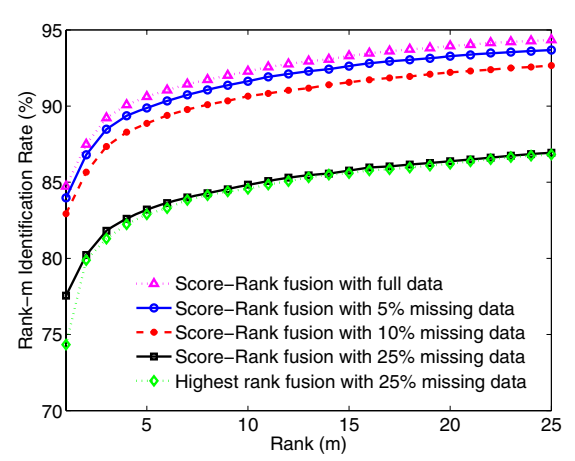

(b)

Fig. 2. Cumulative Match Characteristic (CMC) curve of different fusion rules on the NIST-Face databases under the missing data scenario. (a) Missing templates and (b) missing queries.

From Figure 2, we observe that there is a decrease in the recognition rates with increasing amount of missing data. For this particular database, the reduction in the accuracy is marginal when amount of missing data is less than $10 \%$. However, when the amount of missing data is set to $25 \%$, there is a significant reduction in the accuracy. This decrease in accuracy can be attributed to two reasons. During training, due to missing modalities in the template or query, there are lesser number of match scores available for estimating the marginal score densities. During identification, the identity 
is decided based on fewer evidences compared to the case where there is no missing data. Since the density estimation is sensitive to the number of match scores available during training, the decrease in accuracy depends on the size of the training database. Since the NIST-Face and NIST-Fingerprint databases have a larger number of genuine match scores $(6,000)$, the densities can be reliably estimated even when $10 \%-15 \%$ of the genuine scores are missing. However, the NIST-Multimodal database has only 517 genuine scores. As a result, we observed that even when $10 \%$ of the scores are unavailable, the decrease in the resulting recognition rate is significant.

\section{Conclusions}

Fusion in the multibiometric identification scenario is a critical problem in many largescale practical biometric systems. In this work, we have shown that the likelihood ratiobased score fusion algorithm that was originally designed for the verification mode, can be extended for fusion in the identification scenario. Moreover, we have shown that the likelihood ratios computed based on the match scores can be combined with the rank-based posterior probabilities and the hybrid rank and score level fusion scheme achieves high recognition performance in multibiometric identification systems. The proposed fusion rules can also easily deal with missing data that is commonly encountered multibiometric identification systems without any need for ad-hoc modifications.

\section{References}

1. Federal Bureau of Investigation: Integrated Automated Fingerprint Identification System, http://www.fbi.gov/hq/cjisd/iafis.htm

2. Ross, A., Nandakumar, K., Jain, A.K.: Handbook of Multibiometrics. Springer, Heidelberg (2006)

3. Tulyakov, S., Govindaraju, V.: Combining Matching Scores in Identification Model. In: Proceedings of ICDAR, Seoul, South Korea, August-September 2005, pp. 1151-1155 (2005)

4. Dinerstein, S., Dinerstein, J., Ventura, D.: Robust Multi-Modal Biometric Fusion via Multiple SVMs. In: IEEE Intl. Conf. on Systems, Man and Cybernetics, October 2007, pp. 1530 1535 (2007)

5. Nandakumar, K., Chen, Y., Dass, S.C., Jain, A.K.: Likelihood Ratio Based Biometric Score Fusion. IEEE Trans. on PAMI 30(2), 342-347 (2008)

6. Duda, R.O., Hart, P.E., Stork, D.G.: Pattern Classification. John Wiley \& Sons, Chichester (2001)

7. Ho, T.K., Hull, J.J., Srihari, S.N.: Decision Combination in Multiple Classifier Systems. IEEE Trans. on PAMI 16(1), 66-75 (1994)

8. Melnik, O., Vardi, Y., Zhang, C.H.: Mixed Group Ranks: Preference and Confidence in Classifier Combination. IEEE Trans. on PAMI 26(8), 973-981 (2004)

9. Moon, H., Phillips, P.J.: Computational and Performance Aspects of PCA-based Face Recognition Algorithms. Perception 30(5), 303-321 (2001)

10. Grother, P., Phillips, P.J.: Models of Large Population Recognition Performance. In: Proceedings of CVPR, Washington DC, USA, June/July 2004, vol. 2, pp. 68-75 (2004)

11. Bolle, R., Connell, J., Pankanti, S., Ratha, N., Senior, A.: The Relationship Between the ROC Curve and the CMC. In: Proceedings of Fourth IEEE Workshop on Automatic Identification Advanced Technologies (AutoID), Buffalo, USA, October 2005, pp. 15-20 (2005) 